\title{
Rumor Source Obfuscation on Irregular Trees
}

\author{
Giulia Fanti \\ University of Illinois at \\ Urbana-Champaign \\ fanti@illinois.edu \\ Kannan Ramchandran
University of California,
Berkeley
kannanr@eecs.berkeley.edu
}

\author{
Sewoong Oh \\ University of Illinois at \\ Urbana-Champaign \\ swoh@illinois.edu
}

\author{
Pramod Viswanath \\ University of Illinois at \\ Urbana-Champaign \\ pramodv@illinois.edu
}

\begin{abstract}
Anonymous messaging applications have recently gained popularity as a means for sharing opinions without fear of judgment or repercussion. Messages in these applications propagate anonymously (without authorship metadata) over a network that is typically defined by social connections or physical proximity. However, recent advances in rumor source detection show that the source of such an anonymous message can be inferred by statistical inference attacks. Adaptive diffusion was recently proposed as a solution that achieves optimal source obfuscation over regular trees. However, in real social networks, node degrees differ from node to node, and adaptive diffusion can be significantly sub-optimal. This gap increases as the degrees become more irregular.

In order to quantify this gap, we model the underlying network as coming from standard branching processes with i.i.d. degree distributions. Building upon the analysis techniques from branching processes, we give an analytical characterization of the dependence between the probability of detection achieved by adaptive diffusion and the degree distribution. Further, this analysis provides a key insight: passing a rumor to a friend who has many friends makes the source more ambiguous. This leads to a new family of protocols that we call Preferential Attachment Adaptive Diffusion (PAAD). When messages are propagated according to PAAD, we give both the MAP estimator for finding the source and also an analysis of the probability of detection achieved by this adversary. The analytical results are not directly comparable, since the adversary's observed information has a different distribution under adaptive diffusion than under PAAD. Instead, we present results from numerical experiments that suggest that PAAD achieves a lower probability of detection, at the cost of increased communication for coordination.
\end{abstract}

\section{Keywords}

Anonymous Social Media; Rumor Spreading; Privacy

Permission to make digital or hard copies of all or part of this work for personal or classroom use is granted without fee provided that copies are not made or distributed for profit or commercial advantage and that copies bear this notice and the full citation on the first page. Copyrights for components of this work owned by others than ACM must be honored. Abstracting with credit is permitted. To copy otherwise, or republish, to post on servers or to redistribute to lists, requires prior specific permission and/or a fee. Request permissions from permissions@ acm.org.

SIGMETRICS '16, June 14-18, 2016, Antibes Juan-Les-Pins, France

(C) 2016 ACM. ISBN 978-1-4503-4266-7/16/06 . \$ $\$ 15.00$

DOI: http://dx.doi.org/10.1145/2896377.2901471

\section{INTRODUCTION}

People have a right to share their thoughts without fear of political or economic repercussion. In cyberspace, freedom of expression often depends on a person's ability to remain anonymous to one's family, peers, or even government. Several anonymous messaging apps have emerged and evolved in recent years that allow users to post contents anonymously - first-generation apps like Whisper [1] and Yik Yak 2], second-generation ones like Secret 3] (which is now out of business) and third generation ones like Blind 4. These apps build upon an underlying connectivity network between their users, representing a social graph or a physical proximity graph, for instance. When a user posts a message, the message spreads to the user's neighbors, or 'friends', on the connectivity graph. If a friend approves the message by 'liking' it, the message propagates to the friend's friends, and so forth, spreading over the network.

The centralized architecture of existing anonymous messaging apps makes them vulnerable to deanonymization, since authorship information is stored on central servers. Third parties could access that information via hacking or government subpoena. A distributed architecture instead enables users to propagate messages directly to one another, circumventing centralized storage.

Perhaps surprisingly, even under distributed architectures, the source of a message can still be de-anonymized by global adversaries performing statistical inference. Recent work in rumor source detection shows that the spreading pattern itself reveals a great deal about the true message source 32 . 30]. Existing platforms transmit messages to all neighbors immediately upon approval (e.g. when a user clicks 'like'). There is local randomness in each message's spread due to the time it takes for a user to see the message, and uncertainty regarding whether the user will like the message. This random process is typically modeled by the standard random diffusion process on graphs.

Rumor source detection algorithms, such as those proposed in 32, 30, exploit the inherent symmetry in how random diffusion propagates. In particular, it is shown in 32 that if an adversary, who knows the underlying contact network, observes which nodes received the message at a certain time, the source can be identified with probability bounded away from zero.

Under this vulnerability against statistical inference attacks, a natural question is, "how can a platform designer intervene with the spread of messages, in order to make ru- 
mor source inference difficult?" In other words, how can we add artificial delays on top of the natural human delays to obfuscate the source?

This question was asked in a recent work 14, which proposes a protocol called adaptive diffusion. This protocol protects the author's anonymity against the kinds of global adversaries typically assumed in rumor source detection literature. Adaptive diffusion breaks the symmetry of random diffusion by spreading messages faster in some directions on the underlying graph than others (detailed description in Section 22. It is shown in 14 that adaptive diffusion achieves perfect obfuscation when: (a) the underlying contact network is a regular tree, and (b) the adversary, who knows the underlying contact network, also observes the snapshot of who has seen the message at a certain point in time. A protocol is said to achieve perfect obfuscation, if it successfully hides among all nodes that have seen the message. Precisely, this happens if the probability of the true source being detected is $1 / n$ when $n$ nodes have received the message at the time of the attack.

Although adaptive diffusion achieves the best possible source obfuscation, the protocol design assumes a network that is regular and cycle-free. The assumption of cycle-free (i.e. tree-structured) network can be defended by observing that a message's spread on any connectivity graph will always be a tree embedded in the true connectivity graph; we assume here that that nodes cannot "be infected", or receive the message, more than once. Without loss of generality, a protocol designer can extract a spanning tree from a given social network, and use only those edges in the tree for spreading the messages. All the privacy guarantees we provide will naturally hold. Having more edges on top of this tree only makes it more difficult for the adversary to locate the source.

On the other hand, experimental results (Figures 6 and 7 and also in [14, Figure 8]) suggest that the performance of adaptive diffusion degrades significantly on certain classes of irregular trees.

In this paper, we ask the fundamental question of how the probability of detection depends on the topology of underlying network, when the network is an irregular tree. A precise characterization of the dependency will reveal why adaptive diffusion fails on irregular trees, and provide a guideline for designing novel spreading protocols that improve upon adaptive diffusion.

Model. We follow the setting introduced in 14 for modeling anonymous messaging. At time $t=0$, a single user $v^{*} \in V$ starts to spread a message on a contact network $G=(V, E)$ where users and contacts are represented by nodes and edges, respectively. We assume a discrete-time system and model the delays due to user approval and intermittent network access via a deterministic delay of one time unit. Upon receiving the message, the messaging platform can choose to send the message to any of its neighbors the next time step, or add additional delay and wait. Therefore, if we do not intervene with how the messages are spread, then a message always propagates with a delay of one time unit per hop. If the contact network is an infinite $d$-regular tree, this process spreads to $(d-1)^{T}$ nodes at time $T$, but the source is trivially detected as the center of the snapshot. The validity of this model is discussed in Section 5 .

Adaptive diffusion is introduced in 14 for hiding the source. The key idea is to add appropriate random delays in order to break the symmetry of the spread. This protocol is shown to achieve perfect obfuscation and is described in the next section.

After $T$ time steps, let $V_{T} \subseteq V, G_{T}$, and $N_{T} \triangleq\left|V_{T}\right|$ denote the set of infected nodes, the subgraph of $G$ containing only $V_{T}$, and the number of infected nodes, respectively. At a certain time $T$, an adversary observes the infected subgraph $G_{T}$ (as well as the underlying connectivity graph $G$ ) and produces an estimate $\hat{v}$ of the source $v^{*}$ of the message (with probability of detection $\left.P_{D}=\mathbb{P}\left(\hat{v}=v^{*}\right)\right)$.

There is a tradeoff between hiding the source and increased delay. Assuming a $d$-regular tree, adaptive diffusion spreads to $(d-1)^{T / 2}$ nodes at time $T$, and it is shown to achieve perfect obfuscation in 14 . We say a protocol achieves a perfect obfuscation if the probability of source detection for the maximum likelihood estimator conditioned on $n$ nodes being infected is upper bounded by

$$
\mathbb{P}\left(\hat{v}=v^{*} \mid N_{T}=n\right)=\frac{1}{n}+o\left(\frac{1}{n}\right) .
$$

However, the detection probability can be significantly larger than $1 / n$ when the degrees are not regular. To quantify this gap, we analyze the average probability of detection when the underlying contact network is generated from standard random irregular trees with i.i.d. degrees.

Contributions. We make the following contributions in this paper:

- We quantify the sub-optimality of adaptive diffusion, when the underlying contact network is a random irregular tree generated from i.i.d. degree distribution. We give an exact characterization of the detection probability, where the randomness is due to both the underlying network and also the protocol. In the process of the analyses, we prove a new concentration result for an extremal value on a Galton-Watson tree, which may be of independent interest.

- We give a general expression for the adversary's maximum a posteriori (MAP) detection rule for a broad class of protocols, that are generalizations of adaptive diffusion.

- We introduce a family of protocols that we call preferential attachment adaptive diffusion (PAAD). We characterize the probability of detection for this class of protocols. We present numerical experiments suggesting that PAAD achieves better obfuscation than adaptive diffusion.

Related Work. Most prior research on anonymous communication has focused on anonymous point-to-point communication, leading to the emergence of Tor 12], Freenet [8], Free Haven [11], and Tarzan [17]. In contrast, we study anonymous broadcast messaging. Anonymous broadcast communication has been studied extensively in the context of DC nets 7, 9, 36, 19, 20, 37. Our work differs from this body of work by considering: (a) a different class of solutions, based on statistical spreading models rather than cryptographic encoding, and (b) a tree-structured network, rather than a fully-connected network.

Within the realm of statistical message spreading models, the problem of detecting the origin of an epidemic or 
the source of a rumor has been studied under the diffusion model. Recent advances in $34,33,38,31,16,25,39,28,29$, 27, 15. show that it is possible to identify the source within a few hops with high probability. Drawing an analogy to epidemics, we refer to a person who has received the message as 'infected' and the act of passing the message as 'spreading the infection'. Just as infectious diseases often spread radially about patient zero geographically, under diffusion, the message spreads in a "ball" around the true source over the network. Thus, the author is very close to the center of that ball, making identification easy. Moreover, this is true independent of the size of that ball.

Recently, 26. modeled the rumor spreading and source identification problem as a "hide and seek" game. 26] studied optimal strategies for both the source and adversary under tree networks from a game theoretic perspective, and derived conditions under which a Nash equilibrium exists. In a different context, [5] studies privacy aware network formation games, where actions are adding/removing edges and the reward is measured by the utility of having friends subtracted by the loss in privacy.

Our problem formulation is closely related to the recent work of [14] where adaptive diffusion was presented, and its optimality under regular tree networks was shown. However, [14 does not study the performance of adaptive diffusion under irregular tree networks. In this work, we (a) prove that adaptive diffusion is sub-optimal under irregular trees, (b) characterize the sub-optimality gap, and (c) present a new class of statistical spreading protocols that improves over adaptive diffusion.

Outline. In Section 2, we describe adaptive diffusion protocol introduced in 14. We then present our theoretical analysis of adaptive diffusion over irregular random trees in Section 3 Section 4 describes our proposed preferentialattachment adaptive diffusion algorithm, and demonstrates through simulation that it outperforms regular adaptive diffusion on irregular random trees. We discuss some implications of this work and open questions in Section 5 . We give the proofs of the main results in Section 6 and in the extended version of this paper 13].

\section{ADAPTIVE DIFFUSION}

For completeness, adaptive diffusion is described in full detail in the appendix (see Protocol 1). Adaptive diffusion as introduced in [14 refers to a family of protocols parametrized by $d_{0}$, where $d_{0}$ indicates the degree of the regular tree network for which the protocol is customized. Since we consider irregular trees in this paper, it is not obvious how to choose the parameter $d_{0}$; we set $d_{0}=\infty$ throughout this paper for two reasons: first, this choice is universal, independent of the topology of the underlying graph. Second, it has been suggested via numerical experiments that this choice of $d_{0}$ achieves a performance close to the optimal choice (e.g. Figure 5 in [14]). The downside of this choice is that some nodes in an (infinite tree) network will never receive the message. Henceforth, we refer to adaptive diffusion with the choice of $d_{0}=\infty$ as simply 'adaptive diffusion'. However, it should be noted that in practice, it is preferable to choose a finite $d_{0}$ to ensure that the messages spread in all directions eventually, as we discuss in Section 5

As illustrated in Figure 1, adaptive diffusion ensures that at every even time step the infected subtree is a balanced tree with the true source $v^{*}$ at one of the leaves. At even time $T$, the infected balanced tree has radius $T / 2$ and the center of this sub-tree is called the virtual source, denoted by $v_{T}$. Notice that two time steps are needed to spread the infection from one balanced tree of radius $T / 2$ rooted at $v_{T}$ to another balanced tree of radius $T / 2+1$ rooted at the next virtual source $v_{T+2}$. The odd time steps are intermediate steps, necessary to make such transitions. Since the propagation of the messages is fully described by the dynamics of the virtual sources, adaptive diffusion only needs to provide a (randomized) rule for choosing the virtual source's location at each (even) time.
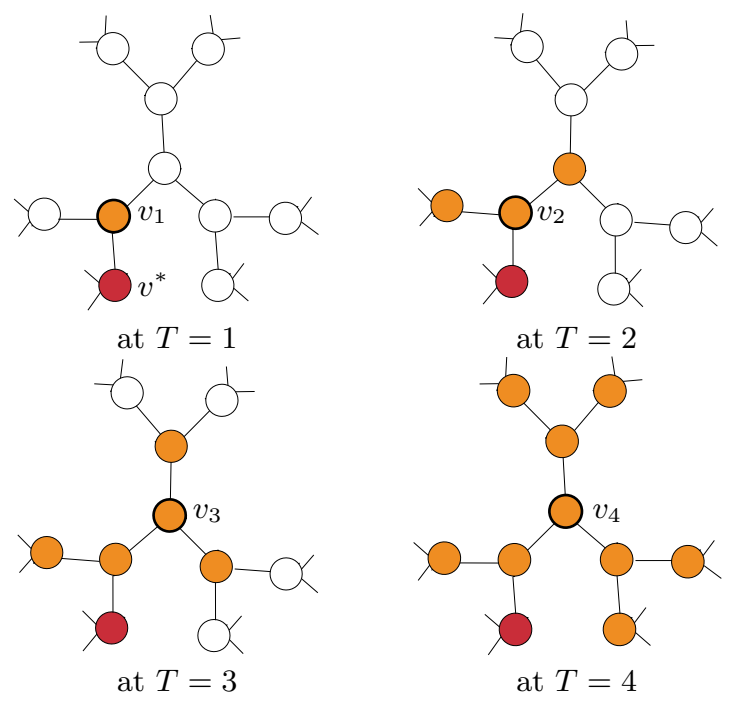

Figure 1: A sample path of adaptive diffusion.

At time $t=0$, the source $v^{*}$ chooses a neighbor to be the next virtual source uniformly at random, and infects it. Let $v_{1}$ denote this new virtual source as shown in Figure 1. The next time step is spent spreading the infection one more hop to appropriate neighbors, in order to maintain the balanced tree of depth one rooted at the new virtual source, which remains $v_{2}=v_{1}$. Our choice of $d_{0}=\infty$ implies that the virtual source never stays the same over consecutive even time steps, so $v_{T+2}$ is always adjacent to $v_{T}$. In other words, in subsequent even time steps, a new virtual source is chosen from one of the current virtual source's neighbor uniformly at random, excluding previous virtual sources. The odd time steps are spent maintaining the balanced infection structure.

\section{ANALYSIS OF ADAPTIVE DIFFUSION}

At a certain time $T$, the adversary attacks and observes the set of infected nodes thus far. Given the snapshot at time $T$, the underlying network, and the knowledge of what protocol is used, the attacker performs statistical inference to detect the source using maximum a posteriori (MAP) estimator. It is proved in [14 that adaptive diffusion achieves (near) perfect obfuscation for regular trees. The key idea is that, by construction, all leaves in the infection are equally likely to have been the source, and there are as many leaf nodes in the boundary as in the interior of the infection.

On irregular trees, adaptive diffusion is known to be suboptimal, and the gap depends on the underlying topology of 
the irregular tree. In order to quantify this gap and characterize the dependence on the underlying tree, we analyze adaptive diffusion on the following model of a random tree and provide rigorous analysis for the average case performance, where the randomness is due to both the underlying contact network as well as the protocol.

We assume adaptive diffusion spreads over a random irregular tree according to a branching process with i.i.d. degrees according to some distribution $D$. Specifically, at time $t=0$, the source $v^{*}$ draws a degree $d_{v^{*}}$ from $D$, and generates $d_{v^{*}}$ child nodes. The source picks one of these neighbors uniformly at random to be the new virtual source. The infection spreads as per adaptive diffusion, and each infected node draws its degree from $D$ generating $D-1$ new (uninfected) children nodes.
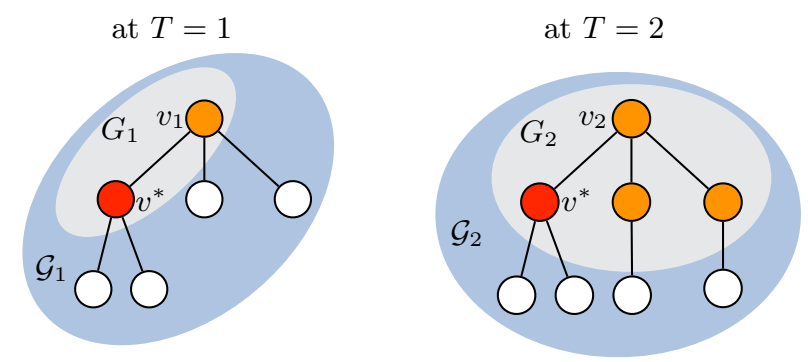

Figure 2: Example of the branching process modeling adaptive diffusion on irregular trees.

We denote the sub-tree of infected nodes as $G_{T}$ and the branching tree generated at time $T$ as $\mathcal{G}_{T}$. The structure of the contact network outside of $\mathcal{G}_{T}$ is independent of $G_{T}$ conditioned on the uninfected neighbors $\partial \mathcal{G}_{T}$, where $\partial \mathcal{G}_{T}$ denotes the leaves of the tree $\mathcal{G}_{T}$. To ensure that the graph grows indefinitely, we assume that the minimum degree of a node is at least two.

\subsection{Probability of Detection Given a Snapshot}

The adversary observes this random process at time $T$, i.e. $\mathcal{G}_{T}$, knowing that the interior $G_{T}$ are the infected nodes, and estimates one of the leaf nodes of $G_{T}$ as an estimate of the true source which started the random process. The following theorem analyzes the probability of detecting the true source for any estimate $\hat{v}$, given a snapshot $\mathcal{G}_{T}$.

THEOREM 3.1. Under the above described random process of adaptive diffusion, an adversary observes the snapshot $\mathcal{G}_{T}$ at an even time $T>0$ and estimates $\hat{v} \in \partial G_{T}$. For any estimator $\hat{v}$, the conditional probability of detection is

$$
\mathbb{P}\left(\hat{v}=v^{*} \mid \mathcal{G}_{T}\right)=\frac{1}{d_{v_{T}}} \prod_{\substack{w \in \phi\left(\hat{v}, v_{T}\right) \\ \backslash\left\{v_{T}, \hat{v}\right\}}} \frac{1}{\left(d_{w}-1\right)},
$$

where $v_{T}$ is the center of $\mathcal{G}_{T}, \phi\left(\hat{v}, v_{T}\right)$ is the (unique) path from $\hat{v}$ to $v_{T}, G_{T}$ is the interior of $\mathcal{G}_{T}$ which is the infected sub-tree, and $\partial G_{T}$ is the set of leaves of $G_{T}$.

A proof is provided in the extended version [13. Intuitively, Equation (2) is the probability that the virtual source starting from $\hat{v}$ ends up at $v_{T}$ (up to some constant factor for normalization). This gives a simple rule for the adversary to achieve the best detection probability by computing the MAP estimate:

$$
\hat{v}_{\mathrm{MAP}}^{(T)} \in \arg \max _{\hat{v}} \mathbb{P}\left(\hat{v}^{(T)}=v^{*} \mid \mathcal{G}_{T}\right) .
$$

COROLlary 3.2. Under the hypotheses of Theorem 3.1. the MAP estimator in (3) can be computed as

$$
\hat{v}_{\mathrm{MAP}}^{(T)}=\arg \min _{v \in \partial G_{T}} \prod_{\substack{w \in \phi\left(v, v_{T}\right) \\ \backslash\left\{v_{T}, v\right\}}}\left(d_{w}-1\right),
$$

achieving a conditional probability of detection

$$
\mathbb{P}\left(\hat{v}_{\mathrm{MAP}}^{(T)}=v^{*} \mid \mathcal{G}_{T}\right)=\max _{v \in \partial G_{T}} \frac{1}{d_{v_{T}} \prod_{\substack{w \in \phi\left(v, v_{T}\right) \\ \backslash\left\{v_{T}, v\right\}}}\left(d_{w}-1\right)}
$$

When applied to regular trees, this recovers known results of [14], which confirms that adaptive diffusion provides strong anonymity guarantees under $d$-regular trees. But more importantly, Corollary 3.2 characterizes how the anonymity guarantee depends on the general topology of the snapshot. We illustrate this in two extreme examples: a regular tree and an extreme example in Figure 3.

For a $d$-regular tree, where all nodes have the same degree, the size of infection at even time $T$ is the number of nodes in a $d$-regular tree of depth $T / 2$ :

$$
N_{T}=\frac{d(d-1)^{T / 2}}{d-2}+\frac{2}{d-2} .
$$

To achieve a perfect obfuscation, we want the probability of detection to decay as $1 / N_{T}$. We can apply Corollary 3.2 to this $d$-regular tree and show the probability of detection is $\left.((d-1) / d)(d-1)^{-T / 2}\right)$, which recovers one of the known results in 14, Proposition 2.2]. This confirms that adaptive diffusion achieves near-perfect obfuscation, up to a small factor of $(d-1) /(d-2)$.

On the other hand, when there exists a path to a leaf node consisting of low-degree nodes, adaptive diffusion can be sub-optimal, and the gap to optimality can be made arbitrarily large. Figure 3 illustrates such an example. This is a tree where all nodes have the same degree $d=5$, except for those nodes along the path from the center $v_{T}$ to a leaf node $v$, including $v_{T}$ and excluding $v$. The center $v_{T}$ has degree two and the nodes in the path have degree three. Hence, the shaded triangles indicate $d$-regular subtrees of appropriate heights. The size of this infection is $N_{T}=\left((d-1)^{T / 2+1} /(d-2)^{2}\right)(1+o(1))$. Ideally, one might hope to achieve a probability of detection that scales as $1 /(d-1)^{T / 2}$. However, Corollary 3.2 shows that the adaptive diffusion achieves probability of detection $1 / 2^{T / 2}$, with the leaf node $v$ achieving this maximum in Equation (5). Hence, there is a multiplicative gap of $((d-1) / 2)^{T / 2}$. By increasing $d$, the gap can be made arbitrarily large. On the other hand, such an extreme topology is rare under the i.i.d tree model.

We want to emphasize that the results in 14 only use elementary proof techniques and only work for regular trees. In comparison, we develop new proof techniques for the combinatorial problem of counting the number of instances that can start from a leaf $v$ and generate a snapshot $\mathcal{G}_{\mathcal{T}}$.

\subsection{Concentration of Probability of Detection}

Depending on the topology, adaptive diffusion can be significantly sub-optimal. A natural question is "what is the 


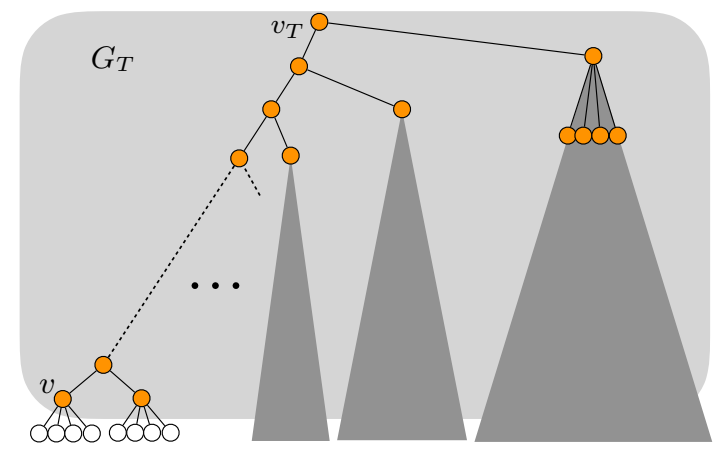

Figure 3: An example of a snapshot emphasizing the suboptimality of the adaptive diffusion.

typical topology of a graph resulting from the random tree model?" Under the model introduced in the beginning of Section 3 we give a concrete answer. Perhaps surprisingly, this typical topology can be characterized by solving a simple convex optimization.

We are interested in the following extremal value

$$
\Lambda_{G_{T}} \equiv d_{v_{T}} \min _{v \in \partial G_{T}} \prod_{\substack{w \in \phi\left(v, v_{T}\right) \\ \backslash\left\{v_{T}, v\right\}}}\left(d_{w}-1\right),
$$

which captures the topology of the snapshot. We want to characterize the typical value of this function over random tree $G_{T}$ resulting from the adaptive diffusion process.

Observe that the distribution of the balanced tree $G_{T}$ follows a simple branching process known as Galton-Watson process. This is because $G_{T}$ resulting from adaptive diffusion has the same distribution, independent of the location of the source $v^{*}$. We consider a given degree distribution $D$. We use $D$ to denote both a random variable and its distribution - the distinction should be clear from context. The random variable $D$ has support $\boldsymbol{f}=\left(f_{1}, \ldots, f_{\eta}\right)$ associated with probability $\boldsymbol{p}=\left(p_{1}, \ldots, p_{\eta}\right)$ such that the degree of node $v$ is i.i.d. with

$$
d_{v}=\left\{\begin{aligned}
f_{1} & \text { with probability } p_{1} \\
\vdots & \vdots \\
f_{\eta} & \text { with probability } p_{\eta},
\end{aligned}\right.
$$

where $2<f_{1}<f_{2}<\cdots<f_{\eta}$ are integers and the positive $p_{i}$ 's sum to one. We also assume $D$ 's support set has at least two elements, i.e., $\eta \geq 2$.

Note that the adaptive diffusion always passes the virtual source token to a uniformly-chosen neighbor. It is straightforward to show that adaptive diffusion starting from a leaf node $v^{*}$ has the same distribution over graphs as the following branching process, denoted $\bar{G}_{T}$ : at time $T=0$ a root node, which we denote as the virtual source $v_{T}$, creates $D$ offspring. At each subsequent even time step, each leaf node in $G_{T}$ creates new offspring independently according to $D-1$ (where we subtract one because each leaf is already connected to its parent). This process is repeated until time step $T$, which generates a random tree $G_{T}$. More precisely, the two branching processes are equal in distribution: $\bar{G}_{T} \stackrel{D}{=} G_{T}$. This can be seen by observing that conditioned on the path of nodes $\phi\left(v^{*}, v_{T}\right)$, the branching processes are identical. Since the node degrees in this path are drawn in- dependently, the path is equally distributed whether it starts from the virtual source $v_{T}$ or the leaf node $v^{*}$.

The following theorem provides a concentration inequality on the extremal quantity $\Lambda_{G_{T}}$, which in turn determines the probability of detection as provided by Corollary 3.2

$$
\mathbb{P}\left(\hat{v}_{\mathrm{MAP}}^{(T)}=v^{*} \mid G_{T}\right)=\frac{1}{\Lambda_{G_{T}}} .
$$

TheOrem 3.3. For an even $T>0$, suppose a random tree $G_{T}$ is generated from the root $v_{T}$ according to the GaltonWatson process with i.i.d. degree distribution D, where $\boldsymbol{f}$ and $\boldsymbol{p}$ are defined as in (8), then the following results hold:

(a) If $p_{1}\left(f_{1}-1\right)>1$, for any positive $\delta>0$, there exists positive constants $C_{D, \delta}$ and $C_{D, \delta}^{\prime}$ that depend only on the degree distribution and the choice of $\delta$ such that

$$
\mathbb{P}\left(\left|\frac{\log \left(\Lambda_{G_{T}}\right)}{T / 2}-\log \left(f_{1}-1\right)\right|>\delta\right) \leq e^{-C_{D, \delta} T},
$$

for an even time $T \geq C_{D, \delta}^{\prime}$.

(b) If $p_{1}\left(f_{1}-1\right)<1$, define the mean number of children:

$$
\mu_{D} \equiv \sum_{i=1}^{\eta} p_{i}\left(f_{i}-1\right)
$$

and the set

$$
\mathcal{R}_{D}=\left\{\boldsymbol{r} \in S_{\eta} \mid \log \left(\mu_{D}\right) \geq D_{\mathrm{KL}}(\boldsymbol{r} \| \boldsymbol{\beta})\right\},
$$

where $S_{\eta}$ denotes the $\eta$-dimensional probability simplex, $D_{\mathrm{KL}}(\cdot \| \cdot)$ denotes Kullback-Leibler divergence, and $\boldsymbol{\beta}$ is a length- $\eta$ probability vector in which $\beta_{i}=p_{i}\left(f_{i}-\right.$ $1) / \mu_{D}$. Further, define $\boldsymbol{r}^{*}$ as follows:

$$
\boldsymbol{r}^{*}=\underset{\boldsymbol{r} \in \mathcal{R}_{D}}{\arg \min }\langle\boldsymbol{r}, \log (\boldsymbol{f}-1)\rangle,
$$

where $\langle\boldsymbol{r}, \log (\boldsymbol{f}-1)\rangle=\sum_{i=1}^{\eta} r_{i} \log \left(f_{i}-1\right)$. Then for any $\delta>0$, there exists positive constants $C_{D, \delta}$ and $C_{D, \delta}^{\prime}$ that only depend on the degree distribution $D$ and the choice of $\delta>0$ such that

$$
\mathbb{P}\left(\left|\frac{\log \left(\Lambda_{G_{T}}\right)}{T / 2}-\left\langle\boldsymbol{r}^{*}, \log (\boldsymbol{f}-1)\right\rangle\right|>\delta\right) \leq e^{-C_{D^{\prime}, \delta} T}
$$

for an even time $T \geq C_{D, \delta}^{\prime}$.

The results in parts $(a)$ and $(b)$ can be merged, in the sense that the solution of $\sqrt{12}$ is $\boldsymbol{r}^{*}=[1,0, \ldots, 0]$ when $p_{1}\left(f_{1}-\right.$ 1) $>1$. A proof of this theorem is provided in Section 6 Putting it together with (9), it follows that the probability of detection concentrates around

$$
-\frac{2}{T} \log \left(\mathbb{P}\left(\hat{v}_{\mathrm{MAP}}^{(T)}=v^{*}\right)\right) \simeq\left\langle\boldsymbol{r}^{*}, \log (\boldsymbol{f}-1)\right\rangle,
$$

in case $(b)$ and around $\log \left(f_{1}-1\right)$ in case $(a)$. Here $\simeq$ indicates concentration for large enough $T$. We want to emphasize that $\boldsymbol{r}^{*}$ can be computed using off-the-shelf optimization tools, since the program in 12 is a convex program of dimension $\eta$. This follows from the fact that the objective is linear in $r$ and the feasible region is convex since KL divergence is convex in $\boldsymbol{r}$.

For example, if $D$ is 3 w.p. 0.7 or 4 w.p. 0.3 , then this falls under case $(a)$. The theorem predicts the probability of detection to decay as $(3-1)^{-T / 2}$. On the other hand, if

$$
D= \begin{cases}2 & \text { with probability } 0.3 \\ 3 & \text { with probability } 0.7\end{cases}
$$


then this falls under case $(b)$ with $\mu_{D}=1.7, \beta_{1}=0.3 / 1.7$, and $\beta_{2}=1.4 / 1.7$. In this case, the exponent is a solution of the following optimization for $\boldsymbol{r}=[r, 1-r]$ :

$$
\begin{array}{cl}
\underset{r \in \mathbb{R}}{\operatorname{minimize}} & r \log 1+(1-r) \log 2 \\
\text { subject to } & r \log \frac{1.7 r}{0.3}+(1-r) \log \frac{1.7(1-r)}{1.4} \leq \log (1.7) \\
& r \in[0,1]
\end{array}
$$

It follows that the optimal solution is $\boldsymbol{r}^{*} \simeq[0.64,0.36]$ and the probability of detection decays as $2^{-0.36(T / 2)}$. Figure 4 confirms this prediction with simulations for these examples.

Theorem 3.3 provides a simple convex program that computes the probability of detection for any degree distribution. For random trees, this quantifies the gap between what adaptive diffusion can guarantee and the perfect obfuscation one desires. We define the rescaled log-multiplicative gap as

$$
\Delta_{D} \equiv \frac{2}{T} \log \frac{\mathbb{P}\left(v_{\mathrm{MAP}}^{(T)}=v^{*}\right)}{1 / \mathbb{E}\left[\left|\partial G_{T}\right|\right]},
$$

where $\left|\partial G_{T}\right|$ is the total number of candidates in a snapshot. It is not difficult to show that $\mathbb{E}\left[\left|\partial G_{T}\right|\right]=\mu_{D}^{T / 2}$, and it follows that $\Delta_{D} \simeq \log \mu_{D}-\left\langle\boldsymbol{r}^{*}, \log (\boldsymbol{f}-1)\right\rangle$. For example, $\Delta_{D}=0$ for regular trees, and $\Delta_{D}=\log _{2} 2.3-\log _{2} 2=0.20$ for the first example under case $(a)$ and $\Delta_{D}=\log _{2} 1.7-0.36=0.41$ for the second example under case $(b)$.

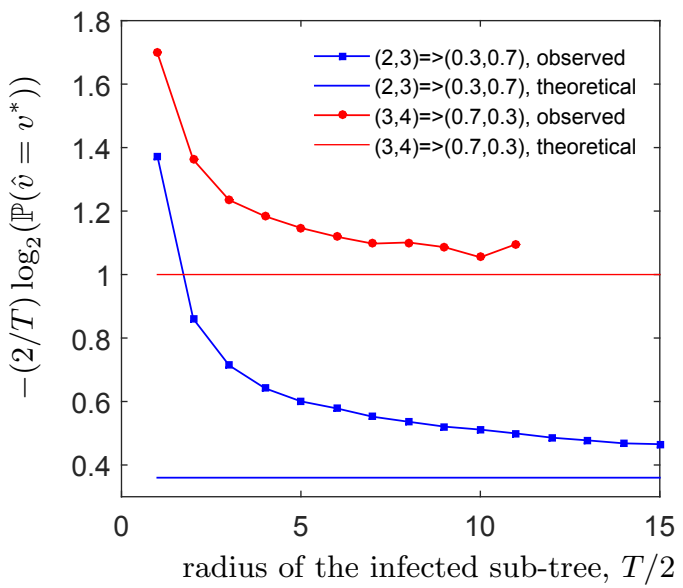

Figure 4: Simulated detection probability converges to the theoretical prediction.

\subsection{Simulation Results}

In Figure 4 we simulate adaptive diffusion and use the MAP estimator in Equation (4) to detect the source. Data points represent the probability of detection, averaged over 10, 000 trials, where the randomness is both in the protocol and the underlying random tree. The horizontal axis denotes successive even time steps of adaptive diffusion-or equivalently, the radius of the infected sub-tree. The notation $(3,4)=>(0.5,0.5)$ in the legend indicates that each node in the tree has degree 3 or 4 , each with probability 0.5 . In this case, we have $p_{1}\left(f_{1}-1\right)>1$, corresponding to case $(a)$ in Theorem 3.3 our analysis predicts that the exponent is $\log _{2}\left(f_{1}-1\right)=1$. This is indicated by a solid red line in Figure 4 For the other distribution with support $\boldsymbol{f}=(2,3)$ with probabilities $\boldsymbol{p}=(0.3,0.7)$, we have $p_{1}\left(f_{1}-1\right)<1$.
This is case $(b)$ in the theorem, and the analysis predicts that the exponent is 0.36 as we computed in the previous section. This is indicated by a solid blue line.

We observe that the empirical exponent $-\log \left(\mathbb{P}\left(\hat{v}=v^{*}\right)\right) /(T / 2)$ converges to the theoretical prediction, albeit slowly. The size of this experiment was limited by computational considerations, since the graph size grows exponentially in time.

\subsection{Sketch of the Proof of Theorem 3.3}

We provide a sketch of a proof of Theorem 3.3 first for case $(a)$. We suppose $\eta=2$ to simplify the notation and highlight the key insights. The random snapshot $G_{T}$ is distributed according to $T$ steps of Galton-Watson process starting from the root node $v_{T}$, with degree distribution $D$. Note that $v_{T}$ is the center of $G_{T}$ and not the source of the message. We want to prove that, with high probability, there exists a path from the root $v_{T}$ to a leaf $v$ which (mostly) consists of nodes with degree $f_{1}$, such that

$$
\min _{v} \prod_{w \in \phi\left(v_{T}, v\right) \backslash\left\{v_{T}, v\right\}}\left(d_{w}-1\right) \simeq\left(f_{1}-1\right)^{T / 2-1},
$$

where $\simeq$ indicates that we allow for vanishing fraction of nodes to deviate from the minimum degree $f_{1}$.

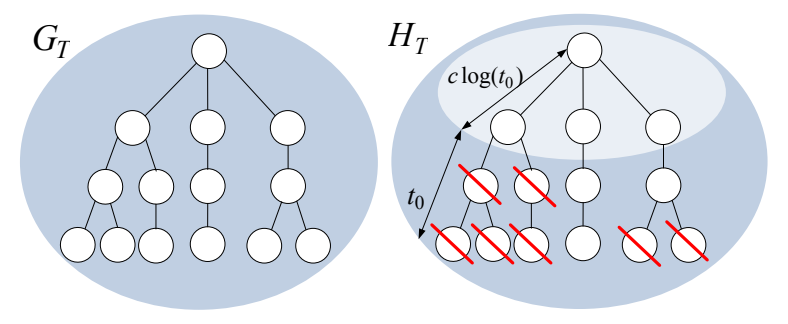

Figure 5: Pruning of a snapshot. We prune all descendants of nodes with degree 3 that are more than $c \log \left(t_{0}\right)$ hops from the root, where $t_{0}+c \log t_{0}=T / 2$.

The main idea is to consider the sub-tree of $G_{T}$ where we remove all nodes with degree $f_{2}$ and also all its descendants. First, if this pruned tree reaches the boundary at $T / 2$, which is referred to as surviving until $T / 2$ time steps, then we know that in the original $G_{T}$ there exists a path from the root to a leaf consisting only of nodes with degree $f_{1}$. Furthermore, The pruned tree is also a Galton-Watson process, but with a different degree distribution:

$$
\tilde{D}=\left\{\begin{aligned}
1 & \text { with probability } p_{2} \\
f_{1} & \text { with probability } p_{1}
\end{aligned}\right.
$$

Under the assumption of case $(a)$ that $\left(f_{1}-1\right) p_{1}>1$, we know from standard analysis of branching processes 22 that this pruned process survives for $T / 2$ time steps with a strictly positive probability for any $T$. By delaying the pruning until an appropriately chosen $O(\log T)$ time steps, we can make this survival probability as large as we want.

In case $(b)$, the same argument proves that the pruned process will not survive, and there will not be a path of minimum degree nodes reaching the boundary. We need to relax the pruning, and allow $r_{2}$ proportion of the nodes in a path to have degree $f_{2}$ for some $r_{2} \in[0,1]$. We search over all possible choices of $\boldsymbol{r}=\left[1-r_{2}, r_{2}\right]$ such that the process 
barely survives while minimizing $\Lambda_{G_{T}}$ :

$$
\begin{array}{cl}
\underset{r_{2} \in[0,1]}{\operatorname{minimize}} & \left(f_{1}-1\right)^{\left(1-r_{2}\right) T / 2}\left(f_{2}-1\right)^{\left(r_{2}\right) T / 2} \\
\text { subject to } & \mathbb{P} \text { (survival) }>0
\end{array}
$$

Note that we have the same objective as in $(12)$, up to a logarithm and scaling. The challenge in analyzing the constraint is that the pruned process is now dynamic, in the sense that we do not fix the number of $f_{2}$ nodes allowed in any path (as we did in case $(a)$, allowing zero $f_{2}$ nodes), but rather let this allowed number grow proportionally to the tree depth. To analyze this dynamically-pruned process, we define a multi-type branching process, where the type of a node encodes the quantity we are interested in, namely the product of degrees in the path from the root. We analyze all possible pruning in this multi-type branching process, and show that it survives if and only if the conditions in 111 is satisfied. The complete proof is provided in Section 6

\section{PREFERENTIAL ATTACHMENT}

Our analysis reveals that adaptive diffusion can be significantly sub-optimal, when the underlying graph degrees are highly irregular. To bridge this gap, we introduce a family of protocols we call Preferential Attachment Adaptive Diffusion (PAAD). We analyze the performance of PAAD and provide numerical simulations showing that PAAD improves over adaptive diffusion when degrees are irregular.

The reason for this gap is that in typical random trees, there are nodes that are significantly more likely to be the source, compared to other typical candidate nodes. To achieve near-perfect obfuscation, we want all candidate nodes to have similar posterior probabilities of being the source. To balance the posterior probabilities of leaf nodes, we suggest passing the virtual source with higher probability to highdegree nodes. We propose a family of protocols based on this idea, and make this intuition precise in Theorem 4.1 .

PAAD is based on adaptive diffusion, but we modify how virtual sources are chosen. We parametrize this family of protocols by a non-negative integer $g$. When a new virtual source is to be chosen, instead of choosing uniformly among its neighbors (except for the previous virtual source), the new virtual source is selected with probability weighted by the size of its $g$-hop neighborhood. Let $\mathcal{N}_{g}(v)$ denote the set of $g$-hop neighbors of node $v$, and let $\mathcal{N}_{g}(v, w)$ denote the same set, removing any nodes $z$ for which $w \in \phi(z, v)$, where $\phi(z, v)$ denotes the path between $z$ and $v$. Then for instance, if $g=1$, then each time the virtual source is passed from $v_{T}$ to $v_{T+2}$, it is passed to a neighbor $w \in \mathcal{N}_{1}\left(v_{T}, v_{T-2}\right)$ with probability proportional to $d_{w}-1$ :

$$
\mathbb{P}\left(v_{T+2}=w\right)=\frac{d_{w}-1}{\sum_{w^{\prime} \in \mathcal{N}_{1}\left(v_{T}, v_{T-2}\right)}\left(d_{w^{\prime}}-1\right)} .
$$

For general $g$, the probability is proportional to the size of the candidate $w$ 's $g$-hop local neighborhood, excluding those in the direction of the current virtual source $v_{T}$. Each virtual source $v_{T}$ chooses the next virtual source as follows: for any node $w \in \mathcal{N}_{1}\left(v_{T}, v_{T-2}\right)$,

$$
P\left(v_{T+2}=w\right)=\frac{\left|\mathcal{N}_{g}\left(w, v_{T}\right)\right|}{\sum_{w^{\prime} \in \mathcal{N}_{1}\left(v_{T}, v_{T-2}\right)}\left|\mathcal{N}_{g}\left(w^{\prime}, v_{T}\right)\right|} .
$$

PAAD encourages the virtual source to traverse high-degree nodes. This balances the posterior probabilities, by strength- ening the probability of leaf nodes whose path contain highdegree nodes, while weakening those with low-degree nodes.

This intuition is made precise in the following theorem, which analyzes the probability of detection for a given snapshot. Define the probability that the sequence of decisions on choosing the virtual sources results in the path from a source $v$ to the current virtual source $v_{T}$ as $Q\left(\mathcal{G}_{T}, v\right) \equiv$ $\prod_{t=1}^{T / 2} \mathbb{P}\left(v_{2 t}=w_{t}\right)$, where

$\phi\left(v, v_{T}\right)=\left(w_{0}=v, w_{1}, w_{2}, \ldots, w_{T / 2-1}, w_{T / 2}=v_{T}\right)$. The specific probability depends on the choice of $g$ and the topology of the underlying tree. Note that the progression of the virtual source now depends on $g$-hop neighborhood, and we therefore define $\mathcal{G}_{T}$ to include the current infected subgraph $G_{T}$ and its $(g+1)$-hop neighborhood.

TheOREM 4.1. Suppose a node $v^{*}$ starts to spread a message at time $t=0$ according to PAAD, where the underlying irregular tree is generated according to the random branching process described in the beginning of Section 3 . At a certain even time $T \geq 0$, an adversary observes the snapshot of the infected subtree $\mathcal{G}_{T}$ and computes a MAP estimate of the source $v^{*}$. Then, the following results hold:

(a) The MAP estimator is

$$
\hat{v}_{\mathrm{MAP}}=\arg \max _{v \in \partial G_{T}} d_{v} Q\left(\mathcal{G}_{T}, v\right)
$$

where $\partial G_{T}$ denotes the leaves of $G_{T}$.

(b) The conditional probability of detection achieved by the $M A P$ estimator is

$$
\mathbb{P}\left(\hat{v}_{\mathrm{MAP}}=v^{*} \mid \mathcal{G}_{T}\right)=\frac{\max _{v \in \partial G_{T}} d_{v} Q\left(\mathcal{G}_{T}, v\right)}{\sum_{w \in \partial G_{T}} d_{w} Q\left(\mathcal{G}_{T}, w\right)}
$$

The proof relies on the techniques developed for Theorem 3.1. and is omitted due to space limitation. The example from Figure 3 illustrates the power of PAAD. For this class of snapshots, it is straightforward to show that under adaptive diffusion, $P_{D}^{A D}=2^{-T / 2}$, whereas under 1-hop PAAD,

$$
P_{D}^{P A A D} \leq \frac{2}{(d-1)^{T / 2-1}-1} .
$$

Notice from these expressions that $P_{D}^{P A A D}$ scales as $(d-$ $1)^{-T / 2}$, which achieves perfect obfuscation, whereas regular adaptive diffusion scales as $2^{-T / 2}$.

This shows that there exist snapshots where PAAD significantly improves over adaptive diffusion. However, such examples are rare under the random tree model, and there are also examples of snapshots where adaptive diffusion can achieve a better obfuscation than PAAD. To complete the analysis, we would like to show the analog of Theorem 3.3 for PAAD. However, the observed snapshot is no longer generated by a standard Galton-Watson branching process, due to the preferential attachment. The analysis techniques developed for Theorem 3.3 do not generalize, and new techniques seem to be needed for a technical analysis. This is outside the scope of this manuscript, but we show simulations suggesting that PAAD improves over adaptive diffusion.

\subsection{Simulation Results}

PAAD requires each virtual source to know some information about its local neighborhood on the contact network; in exchange, we observe empirically that it hides the source 
better than traditional adaptive diffusion. Figure 6 6 shows the probability of detection over graphs with a degree distribution of support $\boldsymbol{f}=(2,5)$ with probability $\boldsymbol{p}=(0.5,0.5)$. The results are averaged over 10,000 realizations of the random graph and the spreading sequence. This plot shows empirically that preferential attachment adaptive diffusion exhibits better hiding properties than regular adaptive diffusion, and that the benefit of preferential attachment increases with the size of the neighborhood considered for preferential attachment (e.g., one-hop vs. two-hop). Notice that our lower bound on probability of detection is $1 /\left|\partial G_{T}\right|$ rather than $1 / N_{T}$, as in 14 ; this is because we constrain the source to always be at one of the leaves of the graph, so $1 /\left|\partial G_{T}\right|$ lower bounds the probability of detection.

Figure 7 computes the ratio of the observed probability of detection to a lower bound on the probability of detection (i.e., $1 /\left|\partial G_{T}\right|$ ), for both adaptive diffusion (AD) and one-hop PAAD. Empirically, we observe that the advantage of PAAD is greater when the degree distribution is more imbalanced (i.e., when $f_{\max }-f_{\min }$ is large).

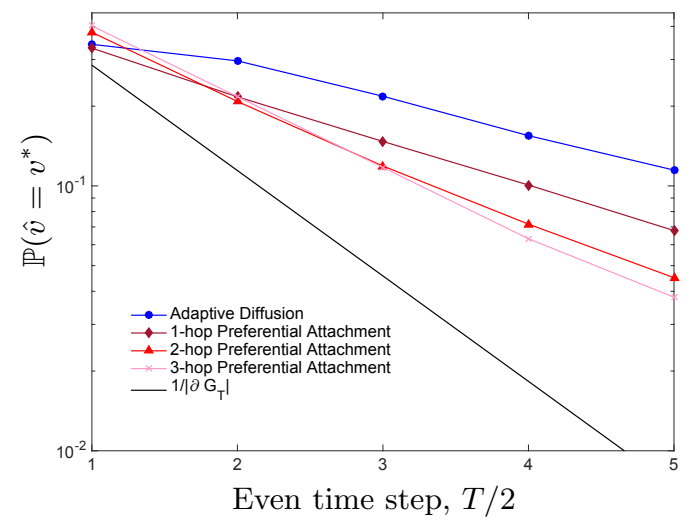

Figure 6: Probability of detection of regular adaptive diffusion compared to 1-, 2-, and 3-hop preferential attachment adaptive diffusion (PAAD).

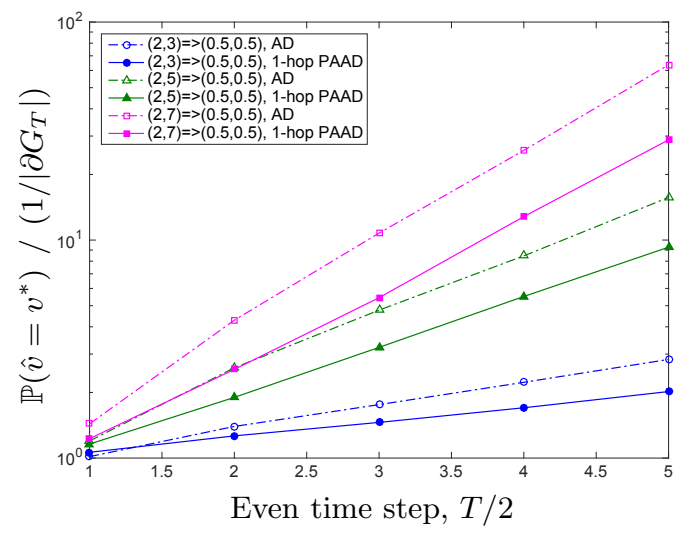

Figure 7: Ratio of observed probability of detection to lowerbound probability of detection, for a range of degree distributions. PAAD has better anonymity properties than regular adaptive diffusion over random, irregular trees.

\section{DISCUSSION}

We characterize how the probability of detection depends on the degree distribution of the underlying random tree, when messages spread as per adaptive diffusion. This suggests a novel family of protocols, we call preferential attachment adaptive diffusion. We analytically calculate the conditional probability of detection of this family of protocols; numerical results suggest improvements over adaptive diffusion. Ideally, we would like to prove an upper bound on the average probability of detection under adaptive diffusion: $\mathbb{P}\left(\hat{v}_{\mathrm{MAP}}^{(T)}=v^{*}\right)=\sum_{\mathcal{G}_{T}} \mathbb{P}\left(\mathcal{G}_{T}\right) \mathbb{P}\left(\hat{v}_{\mathrm{MAP}}^{(T)}=v^{*} \mid \mathcal{G}_{T}\right)$, averaged over all instances of the graphs. We conjecture this has the similar scaling as the concentration result in Theorem 3.3 The challenge is in proving a sharper concentration of $\Lambda_{G_{T}}$.

Proving a result analogous to Theorem 3.3 for PAAD is an interesting research direction, with technical challenges due to the fact that snapshot $G_{T}$ is no longer a Galton-Watson tree. Although the distribution of the snapshot can be modeled by a multi-type branching process, it is an interesting open question how to analyze the typical value of $Q\left(\mathcal{G}_{T}, v\right)$. Similarly, it would be interesting to analyze adaptive diffusion and PAAD when the source is not constrained to be a leaf. This is more practical, in the sense that a natural message spreading should spread to all directions eventually. We believe the techniques developed in this paper can be generalized to handle this more general adaptive diffusion.

A related question is how to give plausible deniability to users that "approve" sensitive messages. Initial investigations exist 18, but technical and algorithmic questions remain, such as fundamental tradeoffs between privacy, spreading rate, and the utility lost, measured by the increased amount of spam in the network.

It has been recently shown that it is difficult to locate multiple sources 35. It might be possible to spread the messages faster than adaptive diffusion and still achieve perfect obfuscation by creating multiple pseudo-sources. This approach sacrifices some social relevance, since the message spreads from remote nodes that may dislike the message.

Finally, recent work explores how to identify the first node in a randomly growing network $6,21,23,24$. Unlike diffusion, the network itself is growing according to some mechanism, e.g., preferential attachment. 6], explores fundamental limits on the size of a candidate set to ensure that the true source or root of the random network is in the candidate set with high probability. A natural question is how to grow such a network to hide the identity of the root.

\section{PROOF OF THEOREM 3.3}

To facilitate the analysis, we consider an alternative random process that generates unlabeled graphs $G_{T}^{\prime}$ according to the same distribution as $G_{T}$ (i.e., the infected, unlabeled subgraph embedded in $U\left(G_{D}^{(T)}\right)$ from the proof of Theorem 3.1. For a given degree distribution $D$ and a stopping time $T$, the new process is defined as a Galton-Watson process in which the set of offsprings at the first time step is drawn from $D$ and the offsprings at subsequent time steps are drawn from $D-1$. At time $t=0$, a given root node $v_{T}$ draws its degree $d_{v_{T}}$ from $D$, and generates $d_{v_{T}}$ child nodes. The resulting tree now has depth 1 . In each subsequent time step, the process traverses each leaf $v$ of the tree, draws its degree from $D$, and generates $d_{v}-1$ children. The random process continues until the tree has depth $T / 2$, since under 
adaptive diffusion, the infected subgraph at even time $T$ has depth $T / 2$. Because the probability of detection in Equation (5) does not depend on the degrees of the leaves of $G_{T}$, the random process stops at depth $T / 2$ rather than $T / 2+1$. We call the output of this random process $G_{T}^{\prime}$. The distribution of $G_{T}^{\prime}$ is identical to the distribution as the previous random process imposed on $G_{T}$, which follows from the proof of Theorem 3.1. We therefore use $G_{T}$ to denote the resulting output in the remainder of this proof.

Distribution $D$ is a multinomial distribution with support $\boldsymbol{f}=\left(f_{1}, \ldots, f_{\eta}\right)$ and probabilities $\boldsymbol{p}=\left(p_{1}, \ldots, p_{\eta}\right)$. Without loss of generality, we assume $2 \leq f_{1}<\ldots<f_{\eta}$. Let $\mu_{D}$ denote the mean number of children generated by $D$ :

$$
\mu_{D}=\sum_{i=1}^{\eta} p_{i}\left(f_{i}-1\right) .
$$

There are two separate classes of distributions, which we deal with as separate cases.

Case 1: When $p_{1}\left(f_{1}-1\right)>1$, we claim that with high probability, there exists a leaf node $v$ in $\partial G_{T}$ such that on the unique path from the root $v_{T}$ to this leaf $v$, all nodes in this path have the minimum degree $f_{1}$, except for a vanishing fraction. To prove this claim, consider a different graph $H_{T}$ derived from $G_{T}$ by pruning large degree nodes:

1. For a fixed, positive $c$, find $t_{0}$ such that $T / 2=t_{0}+$ $c \log \left(t_{0}\right)$.

\section{Initialize $H_{T}$ to be identical to $G_{T}$.}

3. For each node $v \in H_{T}$, if the hop distance $\delta_{H}\left(v, v_{T}\right) \leq$ $c \log \left(t_{0}\right)$, do not modify that node.

4. For each node $v \in H_{T}$, if the hop distance $\delta_{H}\left(v, v_{T}\right)>$ $c \log \left(t_{0}\right)$ and $d_{v}>f_{1}$, prune out all the children of $v$, as well as all their descendants (Figure 5).

We claim that this pruned process survives with high probability. The branching process that generates $H_{T}$ is equivalent to a Galton-Watson process that uses distribution $D-1$ for the first $c \log \left(t_{0}\right)$ generations, and a different degree distribution $D^{\prime}-1$ for the remaining generations; $D^{\prime}$ has support $\boldsymbol{f}^{\prime}=\left(f_{1}, 1\right)$, probability mass $\boldsymbol{p}^{\prime}=\left(p_{1}, 1-p_{1}\right)$, and mean number of children $\mu_{D^{\prime}}=p_{1}\left(f_{1}-1\right)$.

Note that $f_{1} \geq 3$ by the assumption that $p_{1}\left(f_{1}-1\right)>1$. Hence, the inner branching process up to $c \log t_{0}$ has probability of extinction equal to 0 . This means that at a hop distance of $t_{0}$ from $v_{T}$, there are at least $\left(f_{1}-1\right)^{c \log \left(t_{0}\right)}$ nodes. Each of these nodes can be thought of as the source of an independent Galton-Watson branching process with degree distribution $D^{\prime}-1$. By the properties of Galton-Watson branching processes $(22]$, Thm. 6.1), since $\mu_{D^{\prime}}>1$ by assumption, each independent branching process' asymptotic probability of extinction is the unique solution of $g_{D^{\prime}}(s)=s$, for $s \in[0,1)$, where $g_{D^{\prime}}(s)=p_{1} s^{f_{1}-1}+\left(1-p_{1}\right)$ denotes the probability generating function of the distribution $D^{\prime}$. Call this solution $\theta_{D^{\prime}}$. The probability of any individual Galton-Watson process going extinct in the first generation is exactly $1-p_{1}$. It is straightforward to show that $g_{D^{\prime}}(s)$ is convex, and $g_{D^{\prime}}\left(1-p_{1}\right)>1-p_{1}$, which implies that the probability of extinction is nondecreasing over successive generations and upper bounded by $\theta_{D^{\prime}}$. Then for the branching process that generates $H_{T}$, the overall probability of extinction (for a given time $T$ ) is at most $\theta_{D^{\prime}}^{\left(f_{1}-1\right)^{c \log t_{0}}}$. Increasing the constant $c$ therefore decreases the probability of extinction. If there exists at least one leaf at depth $T$ (i.e., extinction did not occur), then there exists at least one path in $H_{T}$ of length $t_{0}-c \log t_{0}$ in which every node (except possibly the final one) has the minimum degree $f_{1}$. This gives

$$
\begin{aligned}
\frac{\log \left(\Lambda_{H_{T}}\right)}{T / 2} & \leq \frac{t_{0} \log \left(f_{1}-1\right)+c \log \left(t_{0}\right) \log \left(f_{\eta}-1\right)}{t_{0}+c \log \left(t_{0}\right)}(16) \\
& \leq \log \left(f_{1}-1\right)+\frac{c \log t_{0}}{t_{0}} \log \frac{f_{\eta}-1}{f_{1}-1}
\end{aligned}
$$

with probability at least $1-\theta_{D^{\prime}}^{\left(f_{1}-1\right)^{c \log t_{0}}}=1-\theta_{D^{\prime}}^{t_{0}^{c \log \left(f_{1}-1\right)}}=$ $1-e^{-C_{D^{\prime}} t_{0}}$, where $C_{D^{\prime}}=\log \left(\theta_{D^{\prime}}\right)$ and the upper bound in 16. comes from assuming all the interior nodes have maximum degree $f_{\eta}$. Since $H_{T}$ is a subgraph of a valid snapshot $G_{T}$, there exists a path in $G_{T}$ from the virtual source $v_{T}$ to a leaf of the tree where the hop distance of the path is exactly $T / 2$, and at least $t_{0}$ nodes have the minimum degree $f_{1}$. Since the second term in 177$)$ is $o\left(t_{0}\right)$, the claim follows. The lower bound $\log \left(\Lambda_{H_{T}}\right) /(T / 2) \geq \log \left(f_{1}-1\right)$ holds by definition. Therefore, for any $\delta>0$, by setting $T$ (and consequently, $t_{0}$ ) large enough, we can make the second term in (17) arbitrarily small. Thus, for $T \geq C_{D, \delta}^{\prime}$, where $C_{D, \delta}^{\prime}$ is a constant that depends only on the degree distribution and $\delta$, the result holds.

Case 2: $\quad$ Consider the case when $p_{1}\left(f_{1}-1\right)<1$. By the properties of Galton-Watson branching processes $(22]$, Thm. 6.1), the previous pruned random process that generated graphs $H_{T}$ goes extinct with probability approaching 1. This implies that with high probability there is no path from the root to a leaf consisting only of min-degree nodes.

Instead, we introduce a Galton-Watson process with multiple types, derived from the original process. Our approach is to assign a numeric type to each node in $G_{T}$ according to the number of non-minimum-degree nodes in the unique path between that node and the virtual source. If a node's path to $v_{T}$ contains too many nodes of high degree, then we prune the node's descendants. The challenge is to choose the smallest pruning threshold that still ensures the pruned tree will survive with high probability. This threshold allows us to precisely characterize $\Lambda_{G_{T}}$ for most of the instances.

To simplify the discussion, we start by considering a special case in which $D$ allows nodes to take only two values of degrees, i.e., $\eta=2$. We extend the results for $\eta=2$ to larger, finite values of $\eta$ in the extended version [13]. With a slight abuse of notation, consider a new random process $H_{T}$ derived from $G_{T}$ by pruning large degree nodes in the following way:

1. For a fixed, positive $c$, find $t_{0}$ s.t. $T / 2=t_{0}+c \log \left(t_{0}\right)$.

2. Initialize $H_{T}$ to be identical to $G_{T}$.

3. For each node $v \in H_{T}$, if the hop distance $\delta_{H}\left(v, v_{T}\right) \leq$ $c \log \left(t_{0}\right)$, do not modify that node, and assign it type 0 .

4. For each node $v \in H_{T}$, if the hop distance $\delta_{H}\left(v, v_{T}\right)>$ $c \log \left(t_{0}\right)$, assign $v$ a type $\xi_{v}$, which is the number of nodes in $\phi(w, v) \backslash\{v\}$ that have the maximum possible degree $f_{2}$, where $w$ is the closest node in $H_{T}$ to $v$ such that $\delta_{H}\left(w, v_{T}\right) \leq$ $c \log \left(t_{0}\right)$ (Figure 8). 


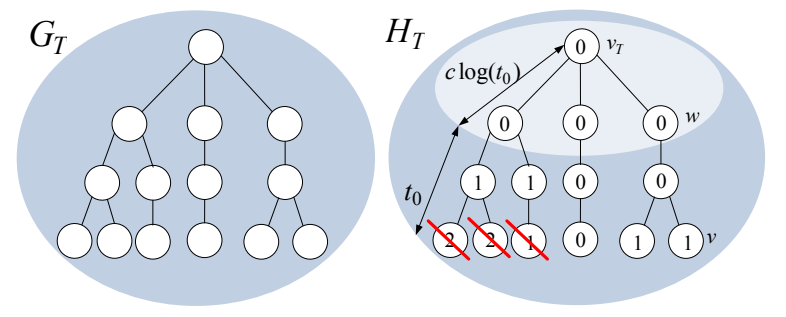

Figure 8: Pruning of a snapshot using multiple types. In this example, the distribution $D$ allows nodes to have degree 2 or 3. We take $t_{0}=2$ and $r=0.5$, so all descendants of nodes with type $r t_{0}=1$ are pruned.

5. Given a threshold $r \in(0,1)$, if a node $v$ has type $\xi_{v} \geq r t_{0}$, prune out all the descendants of $v$. For example, in Figure 8 if $t_{0}=2$ and the threshold is $r=0.5$, we would prune out all descendants of nodes with $\xi_{v} \geq 1$.

We show that for an appropriately-chosen threshold $r$, this pruned tree survives with high probability. By choosing the smallest possible $r$, we ensure that $\Lambda_{H_{T}}$ consists (in all but a vanishing fraction of nodes) of a fraction $r$ nodes with maximum degree, and $(1-r)$ of minimum degree. This allows us to derive the bounds on $\log \left(\Lambda_{H_{T}}\right) /(T / 2)$ stated in the claim, which hold with high probability.

Let $k \equiv r t_{0}$. The process that generates $H_{T}$ is equivalent to a different random branching process that generates nodes in the following manner: set the root's type $\xi_{v_{T}}=0$. At time $t=0$, the root $v_{T}$ draws a number of children according to distribution $D$, and generates $d_{v_{T}}$ children, all type 0 . Each leaf generates type 0 children according to child degree distribution $D-1$ until $c \log \left(t_{0}\right)$ generations have passed. At that point, each leaf $v$ in this branching process (which necessarily has type 0) reproduces as follows: if its type $\xi_{v}>k$, then $v$ does not reproduce. Otherwise, it either generates $\left(f_{1}-1\right)$ children with probability $p_{1}$, each with state $\xi_{v}$, or $\left(f_{2}-1\right)$ children with probability $p_{2}$, each with state $\xi_{v}+1$. This continues for $t_{0}$ generations. Mimicking the notation from Case 1 , we use $D^{\prime}$ to denote the distribution that gives rise to this modified, multi-type random process (in the final $t_{0}$ generations); this is a slight abuse of notation since the branching dynamics are multi-type, not defined by realizations of i.i.d. degree random variables.

Lemma 6.1. Consider a Galton-Watson branching process with child degree distribution $D-1$, where each node has at least one child with probability 1 , and $\mu_{D-1}>1$. Then the number of leaves in generation $t, Z^{(t)}$, satisfies $Z^{(t)} \geq e^{C_{\ell} t}$ with probability at least $1-e^{C_{\ell}^{\prime} t}$, where both $C_{\ell}$ and $\bar{C}_{\ell}^{\prime}$ are constants that depend on the degree distribution.

We omit the proof due to space limitations. The first $c \log \left(t_{0}\right)$ generations ensure that with high probability, we have at least $e^{C_{\ell} \log t_{0}}$ independent multi-type Galton-Watson processes originating from the leaves of the inner subgraph; this follows from Lemma 6.1. Here we have encapsulated the constant $c$ from the first $c \log \left(t_{0}\right)$ generations in the constant $C_{\ell}$. For example, in Figure 8 , there are 3 independent Galton-Watson processes starting at the leaves of the inner subgraph. We wish to choose $r$ such that the expected number of new leaves generated by each of these processes, at each time step, is large enough to ensure that extinction occurs with probability less than one. For brevity, let $\alpha \equiv p_{1}\left(f_{1}-1\right)$ and let $\beta \equiv p_{2}\left(f_{2}-1\right)$. Let $x^{(t)}$ denote the $(k+1)$-dimensional vector of the expected number of leaves generated with each type from 0 to $k$ in generation $t$. This vector evolves according to the following $(k+1) \times(k+1)$ transition matrix $M$ :

$$
x^{(t+1)}=x^{(t)} \underbrace{\left[\begin{array}{ccccc}
\alpha & \beta & & & \\
& & \ddots & & \\
& & \ddots & \alpha & \beta \\
& & & 0
\end{array}\right]}_{M} .
$$

The last row of $M$ is 0 because a node with type $k$ does not reproduce. Since the root of each process always has type 0 , we have $x^{(0)}=\boldsymbol{e}_{1}$, where $\boldsymbol{e}_{1}$ is the indicator vector with a 1 at index 1 and zeros elsewhere. Let $Z^{(t)}$ denote the expected number of new leaves created in generation $t$. This gives

$$
\mathbb{E}\left[Z^{(t)}\right]=\boldsymbol{e}_{1} M^{t} \mathbb{1}_{(k+1)}^{\top},
$$

where $^{\top}$ denotes a transpose, and $\mathbb{1}_{(k+1)}$ is the $(k+1)$ all-ones vector. When $t<k$, this is a simple binomial expansion of $(\alpha+\beta)^{t}$. For $t \geq k$, this is a truncated expansion up to $k$ :

$$
\mathbb{E}\left[Z^{(t)}\right]=\sum_{i=0}^{k}\left(\begin{array}{l}
t \\
i
\end{array}\right) \alpha^{t-i} \beta^{i} .
$$

We seek a necessary and sufficient condition on $r$ for nonextinction, such that $(1 / t) \log \left(\mathbb{E}\left[Z^{(t)}\right]\right)>0$. Consider a binomial random variable $W$ with parameter $\beta /(\alpha+\beta)=\beta / \mu_{D}$ and $t$ trials. Equation 19 implies that for large $t$,

$$
\begin{aligned}
\mathbb{E}\left[Z^{(t)}\right] & =(\alpha+\beta)^{t} \mathbb{P}(W \leq k) . \\
& =\mu_{D}^{t} \exp \left\{-t D_{\mathrm{KL}}\left(r \| \frac{\beta}{\mu_{D}}\right)+o(t)\right\},
\end{aligned}
$$

by Sanov's theorem 10 . Here $D_{\mathrm{KL}}\left(r \| \beta / \mu_{D}\right)$ denotes the Kullback-Leibler divergence between the two Bernoulli distributions defined by $r$ and $\beta / \mu_{D}$, such that $D_{\mathrm{KL}}\left(r \| \beta / \mu_{D}\right)=$ $(1-r) \log \left((1-r) /\left(\alpha / \mu_{D}\right)\right)+r \log \left(r /\left(\beta / \mu_{D}\right)\right)$. We wish to identify the smallest $r$ for which $(1 / t) \log \left(\mathbb{E}\left[Z^{(t)}\right]\right)$ is bounded away from zero. Such an $r$ is a sufficient (and necessary) condition for the multi-type Galton-Watson process to have a probability of extinction less than 1 . To achieve this, we define the following set of $r$ such that Eq. 21) is strictly positive, for some $\epsilon>0$ :

$$
\mathcal{R}_{\alpha, \beta}(\epsilon)=\left\{r \mid \log \left(\mu_{D}\right) \geq D_{\mathrm{KL}}\left(r \| \beta / \mu_{D}\right)+\epsilon\right\},
$$

Suppose we now choose a threshold $r \in \mathcal{R}_{\alpha, \beta}(\epsilon)$. This is the regime where the modified Galton-Watson process with threshold $r$ has a chance for survival. In other words, the probability of extinction $\theta_{D^{\prime}}$ is strictly less than one. Precisely, $\theta_{D^{\prime}}$ is the unique solution to $s=g_{D^{\prime}}(s)$, where $g_{D^{\prime}}(s)$ denotes the probability generating function of the described multi-type Galton-Watson process. Using the same argument as in Case 1, we can construct a process where the probability of extinction is asymptotically zero. Precisely, we modify the pruning process such that we do not prune any leaves in the first $c \log \left(t_{0}\right)$ generations. This ensures that with high probability, there are at least $e^{C_{\ell} \log \left(t_{0}\right)}$ independent multi-type Galton-Watson processes evolving concurrently after time $c \log \left(t_{0}\right)$, each with probability of ex- 
tinction $\theta_{D^{\prime}}$. Hence with probability at least $1-e^{-2 C_{D^{\prime}} t_{0}}$ (for an appropriate choice of a constant $C_{D^{\prime}}$ that only depends on the degree distribution $D^{\prime}$ and the choice of $r$ ), the overall process does not go extinct.

Our goal is to find the choice of $r$ with minimum product of degrees $\log \left(\Lambda_{G_{T}}\right) /(T / 2)$ that survives. We define $r_{1}$ as: $r_{1} \equiv \underset{r \in \mathcal{R}_{\alpha, \beta}(\epsilon)}{\arg \min }(1-r) \log \left(1-f_{1}\right)+r \log \left(1-f_{2}\right)$. Since $\mathcal{R}_{\alpha, \beta}(\epsilon)$ is just an interval and we are minimizing a linear function with a positive slope, the optimal solution is $r_{1}=$ $\inf _{r \in \mathcal{R}_{\alpha, \beta}(\epsilon)} r$. This is a choice that survives with high probability and has the minimum product of degrees. Precisely, with probability at least $1-e^{-C_{D^{\prime}} T}$, where $C_{D^{\prime}}$ depends on $D^{\prime}$ and $\epsilon$, we have that $\frac{\log \left(\Lambda_{G_{T}}\right)}{T / 2} \leq\left\langle\left[1-r_{1}, r_{1}\right], \log (f-\right.$ $1)\rangle+\frac{c \log \left(t_{0}\right)}{t_{0}} \log \left(f_{2}-1\right)$, where we define the standard inner product $\left\langle\left[1-r_{1}, r_{1}\right], \log (\boldsymbol{f}-1)\right\rangle \triangleq\left(1-r_{1}\right) \log \left(f_{1}-1\right)+$ $r_{1} \log \left(f_{2}-1\right)$. It follows that

$$
\begin{aligned}
& \frac{\log \left(\Lambda_{G_{T}}\right)}{T / 2}-\left\langle\left[1-r^{*}, r^{*}\right], \log (f-1)\right\rangle \leq \\
& \left(r_{1}-r^{*}\right) \log \left(\frac{f_{2}-1}{f_{1}-1}\right)+\frac{c \log \left(t_{0}\right)}{t_{0}} \log \left(f_{2}-1\right)
\end{aligned}
$$

By setting $\epsilon$ small enough and $t_{0}$ large enough, we can make this arbitrarily small. For any $\delta>0$, there exists a positive $\epsilon>0$ such that the first term is bounded by $\delta / 2$. Further, recall that $T / 2=c \log \left(t_{0}\right)+t_{0}$. For any choice of $\epsilon$, there exists a $t_{D^{\prime}, \epsilon}$ such that for all $T \geq t_{D^{\prime}, \epsilon}$ the vanishing term in Eq. 21) is smaller than $\epsilon$. For any given $\delta>0$, there exists a positive $t_{D^{\prime}, \delta}$ such that $T \geq t_{D^{\prime}, \delta}$ implies that the second term is upper bounded by $\delta / 2$. Putting everything together (and setting $\epsilon$ small enough for target $\delta$ ), we get

$$
\mathbb{P}\left(\frac{\log \left(\Lambda_{G_{T}}\right)}{T / 2} \geq\left\langle\left[1-r^{*}, r^{*}\right], \log (\boldsymbol{f}-1)\right\rangle+\delta\right) \leq e^{-C_{D^{\prime}, \delta} T}
$$

for all $T \geq C_{D^{\prime}, \delta}^{\prime}$, where $C_{D^{\prime}, \delta}$ and $C_{D^{\prime}, \delta}^{\prime}$ are positive constants that only depend on the degree distribution $D^{\prime}$ and the choice of $\delta>0$.

For the lower bound, we define the following set of $r$ such that Eq. 21] is strictly negative:

$$
\overline{\mathcal{R}}_{\alpha, \beta}(\epsilon)=\left\{r \mid \log \left(\mu_{D}\right) \leq D_{\mathrm{KL}}\left(r \| \beta / \mu_{D}\right)-\epsilon\right\} .
$$

Choosing $r \in \overline{\mathcal{R}}_{\alpha, \beta}(\epsilon)$ causes extinction with probability approaching 1. Explicitly, $\mathbb{P}\left(Z^{(t)} \neq 0\right)$ is the probability of non-extinction at time $t$, and $\mathbb{P}\left(Z^{(t)} \neq 0\right) \leq \mathbb{E}\left[Z^{(t)}\right]$. By Equation 21 , we have $\mathbb{E}\left[Z^{(t)}\right] \leq e^{t\left(\log \left(\mu_{D}\right)-D_{\mathrm{KL}}\left(r \| \beta / \mu_{D}\right)+o(t)\right)}$ where $\log \left(\mu_{D}\right)-D_{\mathrm{KL}}\left(r \| \beta / \mu_{D}\right) \leq-\epsilon$. The probability of extinction is at least $1-\mathbb{E}\left[Z^{(t)}\right] \geq 1-e^{-t(\epsilon+o(t))}$. So defining $r_{2} \equiv \arg \max (1-r) \log \left(1-f_{1}\right)+r \log \left(1-f_{2}\right)$, we have $r \in \overline{\mathcal{R}}_{\alpha, \beta}(\epsilon)$

$\frac{\log \left(\Lambda_{G_{T}}\right)}{T / 2} \geq\left\langle\left[1-r_{2}, r_{2}\right], \log (\boldsymbol{f}-1)\right\rangle+\frac{c \log \left(t_{0}\right)}{t_{0}} \log \left(f_{1}-1\right)$, with probability at least $1-e^{-C_{D^{\prime}, 2} T}$ where $C_{D^{\prime}, 2}$ is again a constant that depends on $D^{\prime}$ and $\epsilon$. It again follows that

$$
\begin{aligned}
& \frac{\log \left(\Lambda_{G_{T}}\right)}{T / 2}-\left\langle\left[1-r^{*}, r^{*}\right], \log (\boldsymbol{f}-1)\right\rangle \geq \\
& \left(r_{2}-r^{*}\right) \log \left(\frac{f_{2}-1}{f_{1}-1}\right)+\frac{c \log \left(t_{0}\right)}{t_{0}} \log \left(f_{1}-1\right),
\end{aligned}
$$

where $r_{2}-r^{*}$ is strictly negative. Again, for any given $\delta>0$, there exists a positive $\epsilon>0$ such that the first term is lower bounded by $-\delta / 2$, and for any choice of $\epsilon$, there exists a $t_{D^{\prime}, \epsilon}$ such that for all $T \geq t_{D^{\prime}, \epsilon}$ the vanishing term in Eq. (21) is smaller than $\epsilon$. Note that this $\epsilon$ might be different from the one used to show the upper bound. We ultimately choose the smaller of the two $\epsilon$ values. For any given $\delta>0$, there exists a positive $t_{D^{\prime}, \delta}$ such that $T \geq t_{D^{\prime}, \delta}$ implies that the second term is lower bounded by $-\bar{\delta} / 2$. Putting everything together (and setting $\epsilon$ small enough for the target $\delta$ ), we get that

$$
\mathbb{P}\left(\frac{\log \left(\Lambda_{G_{T}}\right)}{T / 2} \leq\left\langle\left[1-r^{*}, r^{*}\right], \log (\boldsymbol{f}-1)\right\rangle-\delta\right) \leq e^{-C_{D^{\prime}, \delta} T}
$$

for all $T \geq C_{D^{\prime}, \delta}^{\prime}$, where $C_{D^{\prime}, \delta}$ and $C_{D^{\prime}, \delta}^{\prime}$ are positive constants that only depend on the degree distribution $D^{\prime}$ and the choice of $\delta>0$. This gives the desired result.

The general case for $\eta>2$ (i.e., when the distribution has support greater than two) appears in the extended version [13.

\section{Acknowledgement}

We would like to thank the anonymous reviewers for their helpful comments. This work has been supported by NSF CISE awards CCF-1422278 and CCF-1553452, and SaTC award CNS-1527754.

\section{REFERENCES}

[1] Whisper, 2012. http://whisper.sh.

[2] Yik yak, 2013. http://www.yikyakapp.com/

[3] Secret, 2014. https://www.secret.ly

[4] Team blind, 2015. http://us.teamblind.com/

[5] D. Acemoglu, A. Makhdoumi, A. Malekian, and A. E. Ozdaglar. Privacy-constrained network formation. MIT Department of Economics Working Paper, 2015.

[6] S. Bubeck, L. Devroye, and G. Lugosi. Finding adam in random growing trees. arXiv preprint arXiv:1411.3317, 2014.

[7] D. Chaum. The dining cryptographers problem: Unconditional sender and recipient untraceability. Journal of cryptology, 1(1), 1988.

[8] I. Clarke, O. Sandberg, B. Wiley, and T.W. Hong. Freenet: A distributed anonymous information storage and retrieval system. In Designing Privacy Enhancing Technologies, 2001.

[9] H. Corrigan-Gibbs and B. Ford. Dissent: accountable anonymous group messaging. In CCS. ACM, 2010.

[10] T. M. Cover and J. A. Thomas. Elements of information theory. John Wiley \& Sons, 2012.

[11] R. Dingledine, M.J. Freedman, and D. Molnar. The free haven project: Distributed anonymous storage service. In Designing Privacy Enhancing Technologies, 2001.

[12] R. Dingledine, N. Mathewson, and P. Syverson. Tor: The second-generation onion router. Technical report, DTIC Document, 2004.

[13] G. Fanti, P. Kairouz, S. Oh, K. Ramchandran, and P. Viswanath. Hiding the rumor source. arXiv preprint arXiv:1509.02849, 2015.

[14] G. Fanti, P. Kairouz, S. Oh, and P. Viswanath. Spy vs. spy: Rumor source obfuscation. In SIGMETRICS Perform. Eval. Rev., volume 43, pages 271-284, 2015. 
[15] S. Feizi, K. Duffy, M. Kellis, and M. Medard. Network infusion to infer information sources in networks. 2014.

[16] V. Fioriti and M. Chinnici. Predicting the sources of an outbreak with a spectral technique. arXiv:1211.2333, 2012.

[17] M.J. Freedman and R. Morris. Tarzan: A peer-to-peer anonymizing network layer. In Proc. CCS. ACM, 2002.

[18] G. Giakkoupis, R. Guerraoui, A. Jégou, A. Kermarrec, and N. Mittal. Privacy-conscious information diffusion in social networks. pages 480-496, 2015.

[19] S. Goel, M. Robson, M. Polte, and E. Sirer. Herbivore: A scalable and efficient protocol for anonymous communication. Technical report, 2003.

[20] P. Golle and A. Juels. Dining cryptographers revisited. In Advances in Cryptology-Eurocrypt 2004, 2004.

[21] E. Gwynne and S. Bubeck. Asymptotic behavior of the eden model with positively homogeneous edge weights. arXiv preprint arXiv:1508.05140, 2015.

[22] T. E. Harris. The theory of branching processes. Courier Corporation, 2002.

[23] V. Jog and P. Loh. Persistence of centrality in random growing trees. arXiv preprint arXiv:1511.01975, 2015.

[24] V. Jog and P. Loh. Analysis of centrality in sublinear preferential attachment trees via the $\mathrm{cmj}$ branching process. arXiv preprint arXiv:1601.06448, 2016.

[25] W. Luo, W. Tay, and M. Leng. How to identify an infection source with limited observations. 2013.

[26] W. Luo, W. P. Tay, and M. Leng. Rumor spreading and source identification: A hide and seek game. arXiv preprint arXiv:1504.04796, 2015.

[27] E. A. Meirom, C. Milling, C. Caramanis, S. Mannor, A. Orda, and S. Shakkottai. Localized epidemic detection in networks with overwhelming noise. 2014.

[28] C. Milling, C. Caramanis, S. Mannor, and S. Shakkottai. Network forensics: Random infection vs spreading epidemic. In SIGMETRICS. ACM, 2012.

[29] C. Milling, C. Caramanis, S. Mannor, and S. Shakkottai. Detecting epidemics using highly noisy data. In MobiHoc, pages 177-186, 2013.

[30] P. C. Pinto, P. Thiran, and M. Vetterli. Locating the source of diffusion in large-scale networks. Physical review letters, 109(6):068702, 2012.

[31] B. A. Prakash, J. Vreeken, and C. Faloutsos. Spotting culprits in epidemics: How many and which ones? In ICDM, volume 12, pages 11-20, 2012.

[32] D. Shah and T. Zaman. Detecting sources of computer viruses in networks: theory and experiment. In $A C M$ SIGMETRICS Performance Evaluation Review, volume 38, pages 203-214. ACM, 2010.

[33] D. Shah and T. Zaman. Finding rumor sources on random graphs. arXiv preprint arXiv:1110.6230, 2011.
[34] D. Shah and T. Zaman. Rumors in a network: Who's the culprit? Information Theory, IEEE Transactions on, 57:5163-5181, Aug 2011.

[35] S. Spencer and R. Srikant. On the impossibility of localizing multiple rumor sources in a line graph. ACM SIGMETRICS Performance Evaluation Review, 43(2):66-68, 2015.

[36] J. van den Hooff, D. Lazar, M. Zaharia, and N. Zeldovich. Vuvuzela: Scalable private messaging resistant to traffic analysis. In SOSP. ACM, 2015.

[37] L. von Ahn, A. Bortz, and N.J. Hopper. K-anonymous message transmission. In CCS. ACM, 2003.

[38] Z. Wang, W. Dong, W. Zhang, and C.W. Tan. Rumor source detection with multiple observations: Fundamental limits and algorithms. In $A C M$ SIGMETRICS, 2014.

[39] K. Zhu and L. Ying. A robust information source estimator with sparse observations. arXiv:1309.4846, 2013.

\section{APPENDIX}

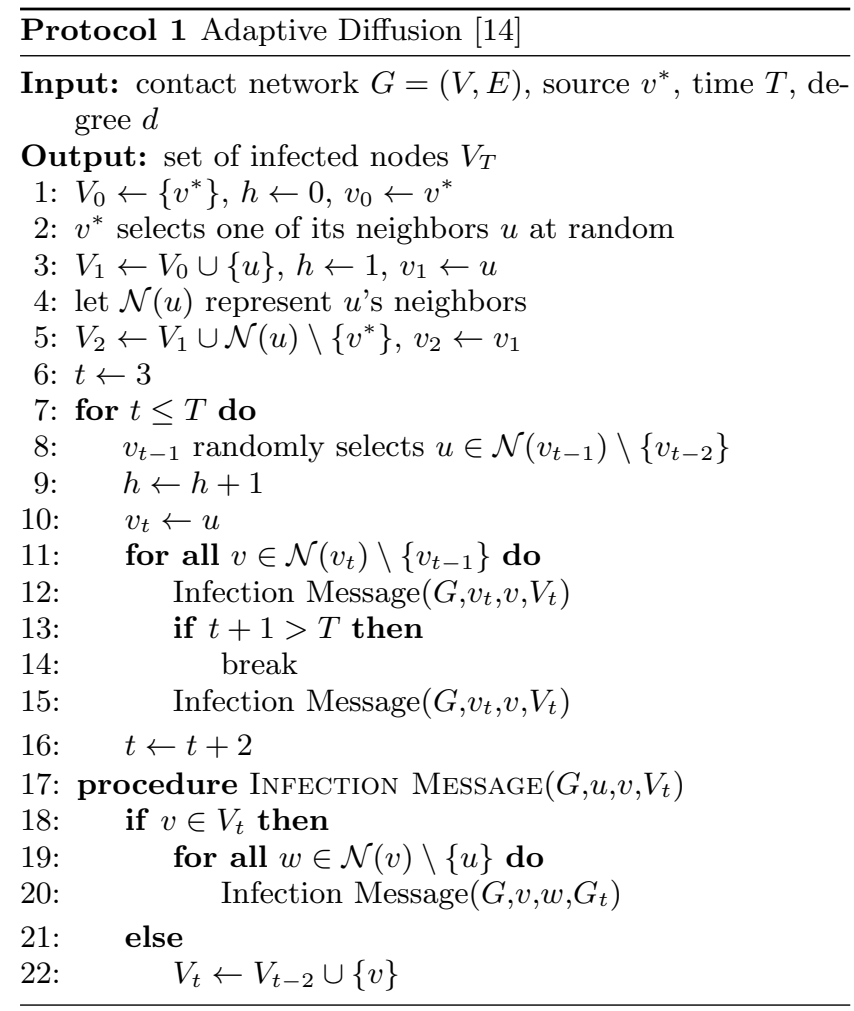

